\title{
Responsividade Materna: Aspectos Biológicos e Variações Culturais
}

\author{
Maternal Responsiveness: Biological Aspects and Cultural Variations
}

\author{
Adriana Ferreira Paes Ribas*a \& Maria Lucia Seidl de Moura ${ }^{b}$ \\ ${ }^{a}$ Universidade Estácio de Sá, Rio de Janeiro, Brasil \\ ${ }^{\mathrm{b}}$ Universidade do Estado do Rio de Janeiro, Rio de Janeiro, Brasil
}

\begin{abstract}
Resumo
Este artigo tem como objetivo discutir criticamente alguns aspectos biológicos e variações culturais relativos ao conceito de responsividade materna. Está subdividido em duas partes. A primeira delas trata da responsividade e seus aspectos biológicos e variações culturais. A segunda apresenta duas grandes tendências de pesquisa sobre responsividade materna e variações culturais. As considerações finais sistematizam os argumentos críticos apresentados e destacam que as iniciativas de investigar este tema devem estar pautadas pelo reconhecimento de que a responsividade materna é uma das características das interações adulto-criança que tem origens e influências múltiplas. Nesse sentido, a sua compreensão deve estar incluída em um sistema amplo de referência que envolva, por exemplo, variáveis biológicas, contextuais, da história da díade e culturais. Palavras-chave: Responsividade materna; aspectos biológicos; interação mãe-bebê; variações culturais.

Abstract

The purpose of this article was to critically discuss some biological aspects and cultural variations in maternal responsiveness. It consists of two parts. The first discusses responsiveness and its biological aspects and cultural variations. The second part presents two major research tendencies in the investigations of cultural variations in maternal responsiveness. Our conclusion presents a brief of the critical arguments and highlights the need to recognize that maternal responsiveness is one of the adult-child interaction characteristics that has multiple origins and influences, from which any investigation in this theme must be based on. As a consequence, those initiatives should be included in a wide reference system that involves, for example, biological, contextual, dyads previous history, and cultural variables.

Keywords: Maternal responsiveness; biological aspects; mother-infant interaction; cultural variations
\end{abstract}

Várias perspectivas em psicologia, como, por exemplo, a teoria do apego (Bowlby, 1984), consideram que as trocas sociais que as crianças estabelecem com seus cuidadores durante a infância podem repercutir sobre o desenvolvimento da própria criança, do ponto de vista cognitivo, emocional e social. Esse é um dos motivos pelos quais, como assinalam diversos autores (Ex. Tamis-LeMonda, Bornstein, Baumwell \& Damast, 1996; Wakschlag \& Hans, 1999), o conceito de responsividade materna tem sido considerado relevante nos estudos sobre desenvolvimento infantil. Embora, como assinalado por Ribas, Seidl de Moura e Ribas Jr. (2003), não haja uma definição única do conceito de responsividade materna, uma possibilidade de definição é a de que a responsividade se caracteriza por comportamentos maternos contingentes, apropriados e imediatamente relacionados aos comportamentos das crianças (Bornstein \& Tamis-LeMonda,

\footnotetext{
* Endereço para correspondência: Rua Maria Amália, 87/ 401, Tijuca, Rio de Janeiro, RJ, 20510-130. E-mails das autoras: aribas@globo.com emlseidl@gmail.com

Este artigo tem origem na tese de doutorado da primeira autora, orientada pela segunda autora, no Programa de Pósgraduação em Psicologia Social da UERJ, em 2004.
}

1997). Esse é um tema amplamente estudado e sobre o qual há uma literatura extensa (vide Ribas, Seidl de Moura \& Ribas Jr., 2003).

A discussão de aspectos naturais e culturais do desenvolvimento, e suas relações com a interação mãe-criança tem recebido a atenção de diversos pesquisadores. Questiona-se em que medida os comportamentos da mãe e da criança podem ser explicados do ponto de vista biológico, e variações culturais podem ser identificadas. Diferentes campos de investigação, como a psicologia, a antropologia, a biologia, e a etnopediatria, uma ciência interdisciplinar iniciada por antropólogos, pediatras e pesquisadores de desenvolvimento infantil (Small, 1999), têm se encarregado de investigar este aspecto, trazendo evidências relevantes.

Este artigo tem como objetivo discutir criticamente aspectos biológicos e variações culturais relacionados à responsividade materna. Organiza-se em duas partes. A primeira trata da responsividade em seus aspectos biológicos e de variações culturais. A segunda parte apresenta duas grandes tendências de pesquisa sobre responsividade materna e variações culturais. Por fim, procura-se sistematizar e discutir os argumentos críticos apresentados. 


\section{Responsividade Materna: Aspectos Biológicos e Variações} Culturais

O aspecto biológico será analisado levando-se em conta principalmente três questões: os sistemas compor tamentais e motivacionais adaptativos, a característica de dependência da criança, e os sistemas de comportamento parental. $\mathrm{O}$ aspecto cultural será abordado levando-se em conta a variedade das práticas parentais e dos comportamentos manifestados pelas crianças.

Quanto aos aspectos biológicos, hipotetiza-se que um conjunto de sistemas comportamentais, provavelmente com valor adaptativo, foi desenvolvido na história evolucionária da espécie humana, tais como aqueles descritos por Bischof (1975) e Bowlby (1984). Os quatro sistemas de comportamento descritos por estes autores são: de apego, de medo, afiliativo e exploratório.

A função do sistema de comportamento de apego é garantir que as crianças se liguem às pessoas com as quais elas poderão obter cuidado e proteção. O sistema de comportamento de medo diz respeito às respostas de evitação. Para Bowlby (1984), este sistema: "aumenta a distância entre pessoas e objetos encarados como ameaçadores” (p. 97). Ambos os sistemas têm valor adaptativo na medida em que aumentam as chances de sobrevivência da criança pela manutenção da proximidade com pessoas capazes de fornecer proteção e da distância daquelas pessoas e objetos considerados ameaçadores.

Deve-se, no entanto, levar em conta ainda que para a espécie humana não seria de fato adaptativo recusar sistematicamente o contato com pessoas com as quais não se tem vínculo, já que ao longo do desenvolvimento estabelecem-se interações com diversas pessoas que não constituem figuras de apego. É exatamente neste ponto que se inclui o sistema afiliativo. Este sistema envolve comportamentos sociais, tais como a vocalização e o sorriso, que são utilizados em interações sociais distais, que não são necessariamente acompanhadas de contato físico. Assim, o comportamento afiliativo permite que a criança entre em contato com o mundo social, e interaja com pessoas que não representam figuras de apego. Por outro lado, também é adaptativo que a criança conheça o mundo que a cerca, e, neste caso, torna-se importante o sistema de comportamento exploratório, a capacidade da criança de conhecer e manipular objetos. Este sistema medeia o contato da criança com o mundo físico.

Além desses sistemas comportamentais, dois sistemas motivacionais básicos presentes desde o nascimento são assinalados por Keller, Lohaus, Volker, Cappenberg, e Chasiotis (1999): um deles para detectar contingências e outro para experimentar calor emocional. Estes sistemas proporcionam à criança, por um lado, a experiência de causalidade e, por outro, a experiência de proximidade emocional com outros. Esses dois sistemas, assim como os anteriormente descritos articulam-se com características de cuidado dos adultos da espécie.

No que se refere à característica de dependência da criança, é preciso considerar o caminho evolucionário da espécie (Bussab 1999; Seidl de Moura \& Ribas, 2004; Small,
1999). O bebê humano é extremamente dependente ao nascer. Não pode ainda, por exemplo, ficar de pé, defender-se, ou encontrar alimento sozinho, o que implica na obrigatoriedade do cuidado e proteção por parte do adulto para garantir a sobrevivência. Essa extrema dependência dos bebês humanos é uma evidência que fortalece a hipótese de que alguns sistemas de cuidado parental fazem de nossa bagagem como espécie (Keller, 2002a; Small, 1999).

O bebê humano, dependente do cuidado do outro, parece nascer muito bem equipado para este contato com aquele que exercerá o papel de seu cuidador. Algumas evidências clássicas sobre pesquisas acerca do desenvolvimento infantil inicial indicam uma predisposição dos bebês para responder seletivamente a eventos sociais (vide Seidl de Moura \& Ribas, 2004 para uma apresentação do «estado da arte» sobre o tema). Por exemplo, os estudos pioneiros de Fantz (1965) sobre a capacidade visual mostraram a seleção de formas e padrões, inclusive a preferência por visualizar rostos humanos. As pesquisas sobre discriminação de sons, indicaram preferências do bebê por estímulos sonoros vinculados à voz humana, e, em especial, a voz da mãe. Estudos sobre discriminação olfativa mostraram também a possibilidade de identificação do cheiro do leite materno, por bebês recém-nascidos, a partir de seis dias de vida. Além dessas capacidades discriminativas e preferências, os bebês humanos apresentam ainda características que atraem a atenção e desencadeiam respostas de cuidado, os kindschenschema, segundo Lorenz (em Seidl de Moura \& Ribas, 2004), e o choro.

Quanto aos sistemas de comportamento parental podese também discuti-los em termos das conseqüências adaptativas da história da espécie. Keller (2002a) descreve cinco sistemas parentais que são utilizados por adultos na interação e cuidado com as crianças.

O sistema de cuidados primários envolve fornecer à criança alimento, higiene e abrigo e seria, provavelmente, o sistema filogeneticamente mais antigo. Este sistema teria a função principal de assegurar a sobrevivência e minimizar o desconforto. Os bebês podem, como conseqüência, vivenciar segurança e confiança na proteção dos outros (em termos de disponibilidade e consistência).

O sistema de contato corporal envolve o carregar o bebê, e o contato físico propiciado por esta prática. Em muitos contextos as crianças são carregadas junto ao corpo de suas mães por muitas horas ao longo do dia. Esta proximidade física levaria, por um lado, a uma redução de perigo e, por outro, a uma oportunidade para a criança experimentar o calor emocional, propiciando o desenvolvimento de vínculo com a mãe e do sentimento de estar relacionado e pertencer (a um núcleo familiar, grupo ou comunidade). Estes dois sistemas não são exclusivos da espécie humana e podem ser observados em outras espécies, como, inclusive, a de roedores.

Os três sistemas descritos a seguir seriam exclusivos da espécie humana. O sistema de estimulação também se baseia em comunicação corporal como o anterior, porém envolve uma atividade exclusivamente diádica, uma vez 
que os adultos (Ex.: pais e mães) se engajam em trocas com seus bebês, estimulando-os através do toque, e do movimento e regulam suas respostas em função das ações dos bebês. Cada resposta é específica para um bebê determinado e não um compor tamento geral. Esse sistema propicia o desenvolvimento motor e da noção do próprio corpo como «agente» no ambiente.

O sistema de estimulação com objetos é também diádico e inclui o ambiente físico que rodeia o bebê e seu cuidador nas trocas entre eles. O adulto chama atenção do bebê para objetos e modula suas respostas pela reação dos mesmos. Esse sistema favoreceria o conhecimento do ambiente, o desenvolvimento cognitivo e, ao mesmo tempo, "o desligamento da dependência de relações sociais" (Keller, 2002a, p. 225).

Finalmente, o sistema de trocas face-a-face caracterizase pelo contato visual mútuo e pelo uso da linguagem. As trocas face-a-face seguem, utilizando a expressão da autora, as regras de "pseudo diálogos" e fornecem à criança experiências de contingência. As respostas "prontas" do adulto para a criança têm relação com a percepção de autoeficácia. Por outro lado, o "calor emocional" que pode ser manifestado nas situações face-a-face é um componente independente que também pode ser vivenciado conjuntamente com a contingência.

Estes sistemas revelariam, segundo Keller (2002a), propensões universais, podendo, entretanto, variar em termos de forma, quantidade e duração em diferentes culturas e supõem, ainda, variações individuais. Bowlby (1984) também considera que, embora os comportamentos humanos sejam variáveis, são encontrados também elementos universais, de importância para a espécie. Uma direção comum é a motivação dos pais para cuidar, alimentar, conhecer e interagir com seus bebês (Keller, 2002a; Small, 1999). Em seus comportamentos dois componentes independentes parecem estar presentes: a contingência e o calor emocional (Keller et al., 1999). Fica clara, assim, a articulação mencionada acima com os sistemas comportamentais e motivacionais dos bebês.

Para Keller (2002a, 2002b) o exercício do papel parental é uma resposta adaptativa e, na mesma direção apontada por Small (1999), um conjunto de comportamentos parentais dirigidos às crianças não são controlados "intencionalmente", e sim fazem parte de um repertório de comportamento que é universal. Bornstein e Cote (2001) supõem a existência de ao menos seis atividades parentais universais: interações de cuidado, interações físicas, interações sociais, interações verbais ou de linguagem, interações didáticas e interações com o uso de materiais.

Como discutido acima, certos comportamentos das crianças e dos adultos que cuidam delas, comuns na espécie humana, e que revelam importante função adaptativa, inclusive a de promover a sobrevivência têm sido identificados e destacados. A responsividade materna tem sido também vinculada a funções adaptativas, como assinala Bornstein (1995). Por isso, pode-se esperar que alguns tipos de responsividade materna sejam similares entre culturas. O exemplo clássico do caráter responsivo do adulto às vocalizações com estresse das crianças pode ilustrar um tipo de compor tamento que possivelmente não difere significativamente entre culturas, uma vez que se relaciona diretamente com a questão da sobrevivência da espécie. Especialmente em comunidades com altas taxas de mortalidade infantil (Ex.: comunidades Aka e Ngandu), a imediata resposta das mães às manifestações de desconfor to, inquietação e choro das crianças, bem como a prática da alimentação ao seio com freqüência e o costume de pegar a criança no colo intensivamente têm sido interpretados como práticas vinculadas à estratégia de prevenção de risco e proteção da criança (Hewlett, Lamb, Shannon, Leyendecker \& Schölmerich, 1998).

As formas de interação entre crianças e pais supõem aspectos biológicos (e também universais) e culturais desde o início da vida. As análises sobre variações culturais têm mostrado, como bem destaca Small (1999), que em alguns casos, o que se julga "natural" é produto da cultura. Esta autora mostra, por exemplo, o quanto se toma como natural a prática ocidental das crianças dormirem sozinhas, quando, na verdade, esse é um padrão culturalmente construído e pouco natural quando se considera o ambiente evolucionário de nossa espécie. As interações e as formas de cuidar das crianças seguem diretrizes culturais que guiam o compor tamento aceitável ou desejável em cada cultura. Essas diretrizes têm uma história cultural, mas, muitas vezes foram consolidadas porque são mais adaptativas para um grupo específico, como por exemplo, as que fundamentam práticas de enrolar os bebês Zinacantecas, restringindo seus movimentos, tal como discutido por Greenfield (2002).

Práticas, atividades ou comportamentos parentais assumem, assim, formas diversas que podem ter funções semelhantes ou diferentes (Bornstein, 1995). Keller (2002b) discute o caráter universal e cultural dos comportamentos envolvidos nas interações entre adultos e crianças. Para ela, existe um repertório de comportamentos da criança e do adulto de natureza universal e que foi produto da evolução. Com base nesse repertório, diferentes grupos culturais irão organizar, selecionar e incentivar diferentes estilos parentais e práticas de cuidado.

Compartilhando essa visão de Keller da relação entre aspectos biológicos, característicos da espécie e culturais, Greenfield (2002) discute as diversas formas pelas quais se dá a mútua definição de cultura e biologia no desenvolvimento. São elas: a cultura reforçando a biologia; apropriando-se da biologia; cultura e biologia adaptando-se mutuamente a para sobrevivência; a cultura selecionando da biologia; a cultura respeitando a biologia; a cultura modelando e atualizando o potencial da biologia. Na verdade, pode-se pensar se é possível separar esses dois componentes. Assim, pressupondo os aspectos biológicos da responsividade materna, na sessão seguinte serão discutidas variações culturais nesse processo. 
Responsividade Materna e Variações Culturais:

\section{Tendências de Pesquisa}

Duas principais tendências na literatura sobre responsividade e os modos de interação adulto-criança em diferentes culturas podem ser identificadas. Um grupo de pesquisas compara amostras americanas e européias com amostras de culturas bastante diferentes em vários aspectos, como a organização social, caráter pré-industrial ou industrial, modos de produção e nível de escolaridade. Em geral esses estudos focalizam o continente africano. Pesquisas realizadas por Harkness e Super (1992) na década de setenta, por exemplo, compararam famílias da comunidade Kipsigs, no Kenya, com famílias de Boston com o objetivo de investigar os diferentes subsistemas que compõem os nichos de desenvolvimento nestas duas culturas. Um segundo conjunto de pesquisas tem buscado comparar entre si culturas urbanas e industrializadas, por exemplo, americanos e europeus. Os estudos realizados por Bornstein et al. (1992) e Bornstein, Maital, Tal e Baras (1995), por exemplo, têm investigado características da responsividade à atividade da criança durante observações naturalísticas em diferentes países (Ex.: Israel, Estados Unidos, França e Japão).

No primeiro grupo mencionado destaca-se o trabalho de Richman, Miller e LeVine (1992), no qual foram investigadas diferenças na responsividade materna entre a comunidade Gusii (rural e predominantemente voltada para a agricultura, com um milhão de pessoas, localizada no sudoeste do Kenya), e moradores de um subúrbio de Boston, observando bebês com idades entre 3 e 4 meses e 9 e 10 meses.

Os resultados desse estudo mostram que mães de ambos os grupos são responsivas aos sinais das crianças, mas apresentam comportamentos que indicam objetivos e estilos diferentes. A responsividade dos Gusii mostra-se basicamente dirigida a acalmar, reduzir o desconforto, atender às manifestações com estresse e tranqüilizar as crianças, não a estimulá-las, nem a engajar-se em interações conversacionais. Por outro lado, a responsividade das mães de Boston tende a engajar as crianças, estimulando a interação pela conversação.

Os padrões de responsividade exibidos pelas mães das duas culturas mostraram-se diferentes em relação aos seguintes comportamentos da criança: vocalização sem estresse, olhar e chorar aos 3 e 4 meses e 9-10 meses de idade. As mães Gusii mais freqüentemente respondem a todos os comportamentos das crianças, nas duas idades observadas, tocando-as e realizando outros contatos físicos. As mães de Boston respondem mais freqüentemente ao olhar e ao choro das crianças aos 3 e 4 meses, pegando ou tocando, mas aos 9-10 meses respondem mais freqüentemente aos três comportamentos citados, falando e olhando para a criança. Os resultados são discutidos pelos autores no sentido de que a freqüência maior de falar e olhar em resposta à vocalização em ambas as idades, pelas mães de Boston revela, possivelmente, que estas mães tratam as vocalizações de seus bebês como uma fala inicial e criam as chamadas "protoconversações". O mesmo não ocorre para as mães Gusii.
Uma explicação para isso, segundo os autores, é que as mães Gusii vêem-se como tendo a função de proteger seus bebês, não de brincar com eles ou educá-los. Do ponto de vista delas, a excitação emocional deve ser evitada e a comunicação verbal só deverá ser estimulada quando a criança for capaz de falar (Richman, Miller \& LeVine, 1992).

Variações nos objetivos e estilos da responsividade materna podem ser interpretadas de uma variedade de maneiras. Entretanto, pode-se levar em conta as diferenças de tradições culturais e condições socioeconômicas dos dois grupos. Para isso pode ser útil a noção de nichos de desenvolvimento (Harkness \& Super, 1994). Essa noção, se constitui em um sistema composto por três subsistemas (ambiente físico e social, práticas de cuidado culturalmente reguladas e psicologia dos cuidadores) que relacionam-se dinamicamente, e destaca muito bem a relação entre diferentes componentes do ambiente ecológico da criança.

A discussão crítica e a pesquisa sobre etnoteorias parentais (a psicologia dos cuidadores) apresentadas por Harkness e Super (1992) são relevantes para a compreensão de como as crenças parentais contribuem para a organização das atividades cotidianas das crianças e como se expressam nas práticas de cuidado. No exemplo citado anteriormente, relativo às mães Gusii, os modos de agir e as crenças parentais mostram-se claramente imbricados. Os autores destacam que as mulheres da comunidade, especialmente as mais velhas, tendem a ridicularizar a idéia de falar com crianças antes que elas sejam capazes de falar.

As mães Gusii são fisicamente responsivas, particularmente ao choro e não tanto à vocalização sem estresse; as mães de Boston são predominantemente verbais e visualmente responsivas, tanto à vocalização quanto ao choro. Estas diferenças culturais são evidentes aos 3 ou 4 meses, porém mais pronunciadas aos 9 e 10 meses, parcialmente, por causa da maior distância física que as mães de Boston colocam entre elas e os bebês, enquanto os Gusii mantêm um padrão de proximidade e contato tátil.

A amostra pequena utilizada nesta pesquisa, aspecto ressaltado pelos próprios autores, não permite, segundo eles, explicações definitivas sobre as diferenças culturais, mas seus resultados trazem elementos relevantes do ponto de vista não só da investigação da responsividade nas interações mãe-bebê, mas da própria avaliação que as mães Gusii fazem sobre seu papel enquanto mães e como vêem o desenvolvimento infantil.

Os mesmos autores, Richman, Miller e LeVine (1992), observaram mães e bebês no México, na cidade de Cuernavaca e os achados sobre responsividade mostraram-se similares aos das mães de Boston. A diferença encontrada entre essas mães foi que para as mães de Boston, as freqüências de falar e olhar, em resposta às crianças, foram muito similares. No México, o olhar materno foi uma resposta muito mais freqüente do que a fala materna.

As pesquisas que comparam grupos africanos a outros grupos culturais são criticadas por Hewlett et al. (1998). Para eles, de modo geral, as pesquisas têm indicado que as práticas de cuidado variam pouco entre as comunidades 
africanas. Estes autores entendem que esses resultados podem, em parte, ser atribuídos ao fato de que foram estudados grupos africanos muito semelhantes entre si, tais como os Kipsigs (Harkness \& Super, 1992) e os !Kung (Hewlett et al., 1998).

Realmente, uma tendência à simplificação pode ser observada na literatura, na medida em que se nomeia como «grupos africanos» diferentes comunidades com características próprias, deixando de considerar a diversidade cultural no mesmo continente e dentro do mesmo país. De certa forma, os "africanos" e também os "japoneses" foram muitas vezes considerados grupos culturais homogêneos, sendo comparados com grupos ocidentais, o que não é apropriado. As investigações da responsividade em diferentes culturas devem levar em conta esta discussão, e evitar considerar como culturas únicas, sem variedade interna, grupos culturais diversificados e complexos.

É isso que é feito por Hewlett et al. (1998) por exemplo, quando focalizaram justamente a variabilidade nos cuidados infantis e nas formas de interagir com crianças em duas comunidades tradicionais na África, os Aka e os Ngandu. Os Aka são caçadores-coletores e mudam seu acampamento várias vezes por ano, os Ngandu são agricultores. Ambas as comunidades vivem na mesma floresta tropical, têm o mínimo de estratificação social, têm índices de mortalidade e fertilidade similares, mas têm formas distintas de produção, de relações entre os sexos e de padrões de cuidado de crianças.

Os resultados da comparação mostraram-se muito instigantes para a discussão sobre diferenças culturais. As interações entre os Aka eram marcadas por maior proximidade. As crianças eram carregadas junto com suas mães quase que todo o tempo e alimentadas com maior freqüência e por mais tempo. Isto pode explicar porque estas crianças dormiam e cochilavam mais. Os autores destacam que esta forma de ser cuidado pode incrementar a capacidade de comunicação não-verbal, já que envolve proximidade física, movimentos corporais, batimentos cardíacos, sons e cheiros. Por outro lado, os Ngandu que são carregados em mais ou menos metade das ocasiões, comparativamente aos Aka, parecem depender mais de formas distais de comunicação. Os Ngandu vocalizavam e estimulavam mais as crianças. As crianças Ngandu, por sua vez, tendiam mais a responder sorrindo e vocalizando, além de mostrar mais inquietação e choro.

Outro aspecto que merece destaque nas comparações entre os Aka e os Ngandu é o que se refere à tendência de atender prontamente às necessidades da criança para garantir a sobrevivência, que tem sido vista como especialmente importante em comunidades com altas taxas de mortalidade. Neste caso, as duas comunidades têm igualmente altas taxas de mortalidade e ainda assim mostram diferentes práticas em relação ao atendimento das necessidades das crianças. Os Aka atendem mais prontamente às manifestações de estresse do que os Ngandu.

Para explicar diferenças entre os grupos, Hewlett et al. (1998) apontam o caráter nômade dos Aka, que faz com que tendam a perceber mais riscos do que os Ngandu que são sedentários. Os Aka carregam mais as crianças, possivelmente para evitar potenciais perigos vinculados à mudança constante de ambiente, que ocorre de quatro a 18 vezes por ano. Outro aspecto que pode explicar o contraste das práticas de cuidado é o das diferenças entre os contextos em que vivem. Na comunidade Aka, entre 25 a 35 pessoas compartilham o espaço correspondente a uma sala de jantar e de estar nos Estados Unidos, e o engajamento em atividades cooperativas é freqüente. Os Ngandu têm uma cooperação mais limitada. Para os padrões europeus e americanos, não são socialmente isolados, mas em comparação com os Aka, seus contatos sociais com outros adultos são menos freqüentes e mais curtos em duração.

Outros dois pontos são destacados. Em primeiro lugar, que os Aka, por terem práticas compartilhadas de colher e caçar, podem estar menos preocupados com a obtenção do alimento e mais dispostos a investir no cuidado das crianças. Segundo, que, possivelmente, o foco dos Aka no retorno imediato e não na gratificação futura pode fazer com que eles estejam mais dispostos a acomodar e suprir as demandas de suas crianças por gratificação.

Os autores finalizam a discussão procurando integrar o estilo de cuidado com o modo de vida da comunidade Aka. $\mathrm{O}$ «nicho» deste grupo é marcado por uma integração de pessoas que compartilham e cooperam em muitas atividades. Talvez, segundo Hewlett et al. (1998), como resultado, as suas práticas de cuidado de crianças sejam caracterizadas por carregar quase continuamente as crianças, por um cuidado freqüente e responsividade pronta, especialmente a sinais de estresse. Vê-se claramente, portanto, como duas comunidades próximas geograficamente constituem "nichos de desenvolvimento" diferenciados e não podem ser reduzidas a um rótulo como, como de "africanas", por exemplo.

Na segunda tendência de estudos, que compara culturas urbanas industrializadas, destacam-se as pesquisas realizadas por Bornstein e colaboradores em diferentes países (Ex.: Bornstein, Maital, Tal \& Baras, 1995). Os resultados de uma pesquisa comparativa de três países: Estados Unidos, Japão e França (Bornstein et al., 1992) indicam que nas três culturas as crianças se comportam de modo similar em termos dos comportamentos observados e do caráter mútuo e apropriado das interações. As mães respondem às explorações do ambiente por parte das crianças com encorajamento para o ambiente, às vocalizações sem estresse com imitação, e às vocalizações com estresse com cuidado. Diferenças na responsividade materna entre culturas ocorreram em relação a olhar e vocalizar por parte das crianças e em relação às mães, em termos de enfatizar as interações diádicas ou extradiádicas.

Bornstein (1995) procura integrar os achados de suas pesquisas com o modelo de forma e função. O autor ilustra a relação "mesma forma - mesma função" ao relatar que pesquisas envolvendo mães de diferentes culturas (Bornstein et al., 1992) mostram poucas diferenças no que diz respeito a vocalizações com ou sem estresse das crianças. Mães na França, Japão e Estados Unidos, por exemplo, respondem às vocalizações com estresse de suas crian- 
ças predominantemente cuidando e respondem às vocalizações sem estresse predominantemente pela imitação vocal.

Diferentes formas e diferentes funções são ilustradas quando o autor comenta que as mães norte-americanas e japonesas são diferentes na forma de reagir e de estimular a atenção das crianças. Mães japonesas respondem mais às orientações sociais, enfatizam a responsividade orientada para a díade. As mães americanas enfatizam a responsividade orientada para o ambiente, pela incorporação do mundo de fora da díade em suas interações. Esta comparação pode ser vista como uma relação de diferente forma e diferente função: diferentes expressões de responsividade revelariam que as mães americanas e japonesas estariam estimulando uma orientação respectivamente individualista e coletivista no desenvolvimento (Bornstein et al., 1992). Por outro lado, esse mesmo resultado poderia ser discutido como uma mesma função estaria sendo cumprida de diferentes formas, já que nos dois casos a responsividade materna serve a mesma função de promover o ajustamento em termos de valores culturais predominantes. Aqui é pertinente a consideração de Ogbu (1981), sobre a teoria nativa de sucesso como um elemento que influencia os valores e práticas de cuidado com as crianças, já que os cuidadores tentam preparar as crianças para seguir orientações desejadas e esperadas por cada cultura.

Nas comparações entre os grupos urbanos e industrializados, um aspecto que merece atenção é o da relação entre responsividade materna, status socioeconômico (SES) da família e escolaridade da mãe. Como discutido por Ribas, Seidl de Moura, Gomes, Soares e Bornstein (2003), dos fatores que habitualmente são utilizados para avaliar o status socioeconômico (Ex.: escolaridade, prestígio ocupacional, renda), a escolaridade parece o componente mais importante a ser considerado.

Um número ainda reduzido de pesquisas tem investigado relações entre responsividade materna, sEs e escolaridade. Algumas pesquisas consultadas relatam não ter encontrado relação entre escolaridade da mãe e responsividade materna. Por exemplo, Bornstein e Tamis-LeMonda (1989), em uma pesquisa com 52 mães americanas e seus bebês de 5 meses, Borsntein e Tamis-LeMonda (1997) com uma amostra de 36 mães americanas e seus bebês de 5 meses, Van Egeren, Barratt e Roach (2001), com uma amostra de 150 mães americanas com bebês de 4 meses e Ribas (2004) com uma amostra de 30 mães brasileiras e bebês de 5 meses não identificaram relação entre responsividade e escolaridade da mãe.

Em contraste, duas outras pesquisas apontam relação entre esses dois componentes que vêm sendo discutidos. Goldberg, Lojkasek, Gartner e Corter (1989) com uma amostra de 38 díades de mães americanas e bebês prétermo de 3, 6 e 9 meses, relataram correlação entre escolaridade materna e responsividade. Richamn, Miller e LeVine (1992), com amostra de 72 mães (americanas e mexicanas) e bebês de 5 e 10 meses, também apontaram correlações entre escolaridade materna e responsividade verbal das mães em resposta ao olhar dos bebês e à vocalização dos bebês. Os autores discutem que, possivelmente, mais anos na escola tem relação com a habilidade verbal das mães.

As divergências observadas nas pesquisas sobre a relação entre escolaridade da mãe e responsividade indicam a necessidade de outras investigações nesse sentido. Este seria mais um aspecto que merece a atenção e discussão crítica dos pesquisadores na área.

\section{Considerações Finais: Responsividade Materna, um Olhar Crítico sobre a Discussão entre o Biológico e o Cultural}

Os vários aspectos discutidos nas partes 1 e 2 do artigo procuraram evidenciar a necessidade de um olhar crítico dos pesquisadores no sentido de tentar integrar diferentes dimensões presentes no conceito de responsividade materna. Nessa parte do trabalho serão organizados, de modo mais sistemático, alguns dos argumentos críticos que as autoras consideram relevantes para essa discussão.

O ponto mais geral de discussão crítica é, por um lado, reconhecer a necessidade e a possibilidade de compreender a responsividade como tendo, provavelmente, uma função adaptativa. Por isso, admitir o seu componente biológico é fundamental. Por outro lado, reconhecer que a responsividade se expressa de modo diferenciado dependendo, por exemplo, das práticas do grupo em termos dos cuidados com crianças, dos estilos parentais, e dos objetivos dos pais ao interagirem com as crianças. Essa integração das duas dimensões parece ser viável e pode ser sustentada com a contribuição de diferentes autores (Ex.: Bornstein, 1995; Bowlby, 1984; Keller, 1998; 2002a; Keller et al., 1999; Small, 1999).

Essa visão da responsividade materna como sendo ao mesmo tempo explicada por componentes biológicos e culturais faz com que haja um maior cuidado ao se discutir e julgar elementos "naturais" e "culturais" das interações mãe-bebê. A distinção entre o natural e o cultural não parece, na verdade, fazer muito sentido e, as duas dimensões estão presentes simultaneamente, muitas vezes não podendo ser diferenciadas. A idéia da relação entre formas e funções, discutida por Bornstein (1995), colabora de modo substantivo para esse olhar crítico, já que ressalta que as práticas ou atividades parentais podem assumir formas e funções diferentes, ainda que se baseiem em algum componente universal.

Entende-se que a responsividade materna, como um aspecto da interação mãe-criança, tem elementos básicos biológicos, mas ganha sentido nas tradições, nas práticas, e nas condições socioeconômicas dos grupos nos quais é estudada. Por isso, afirma-se que a investigação sobre responsividade materna pode ser beneficiada ao adotar uma visão crítica sobre os diferentes componentes do ambiente ecológico da mãe e da criança. Assim, a noção de nichos de desenvolvimento (Harkness \& Super, 1994) e a pesquisa sobre etnoteorias parentais podem ser proveitosas (Harkness \& Super, 1992).

Outro aspecto que deve merecer o olhar crítico dos pesquisadores é a tendência à simplificação que pode ser observada na literatura. Nesse sentido, comparações 
interculturais são desejáveis e necessárias para que grupos complexos e heterogêneos não sejam tratados como uniformes e representativos de uma realidade única (Ex.: os africanos).

As pesquisas já realizadas sobre responsividade em diferentes contextos culturais certamente têm contribuído para que se conheça um pouco melhor os nichos de desenvolvimento infantil. Entretanto, parece que tais iniciativas ainda se mostram insuficientes, pois, segundo Bornstein e Lamb (1992), apesar de pesquisas que investigam diferentes países, grande parte do conhecimento sobre desenvolvimento infantil e interação mãe-criança deriva de estudos realizados com amostras de crianças e famílias de classe média urbana dos Estados Unidos. Grande parte, portanto, das crianças do mundo não estão devidamente representadas na literatura.

No caso da pesquisa nacional, há uma carência de estudos sobre responsividade materna (ver Ribas, Seidl de Moura \& Ribas, 2003) e a necessidade de suprir essa lacuna. Deve-se destacar ainda a necessidade de estudos interculturais no nosso país, por exemplo, sobre padrões de cuidado parental e responsividade em diferentes regiões, em contextos urbanos e rurais, com amostras de diferentes níveis de escolaridade, etc.

Em síntese, como discutido por Ribas, Seidl de Moura e Ribas (2003) iniciativas de investigar a responsividade materna de modo integrado ao estudo das crenças parentais, das práticas de cuidado, do conhecimento do contexto de desenvolvimento das crianças, do nível socioeconômico da família, enfim, dos diversos elementos que constituem os nichos de desenvolvimento da criança parecem muito necessárias. Neste sentido, uma articulação com a abordagem sociocultural pode ser proveitosa. Entretanto, investigar elementos do contexto, certamente não exclui considerar, ao mesmo tempo, componentes biológicos envolvidos nas trocas entre mães e bebês e, especificamente, na responsividade materna.

Finalmente, pode-se afirmar que as iniciativas de investigar este tema devem estar pautadas pelo reconhecimento de que a responsividade materna, com suas múltiplas origens e influências, é uma das características das interações adulto-criança, e sua compreensão deve estar incluída em sistema amplo de referências que envolva níveis diversos de análise.

\section{Referências}

Bischof, N. (1975). A systems approach toward the functional connections of attachment and fear. Child Development, 46, 801-817.

Bornstein, M. H. (1995). Form and function: Implications for studies of culture and human development. Culture $\mathcal{E}^{\circ}$ Psychology, 1, 123-137.

Bornstein, M. H., \& Cote, L. R. (2001). Mother-infant interaction and acculturation: I. Behavioural comparisons in Japanese American and South American families. International Journal of Behavioral Development, 25, 549-563.
Bornstein, M. H., \& Lamb, M. E. (1992). Development in infancy: An introduction. New York: McGraw-Hill.

Bornstein, M. H., Maital, S. L., Tal, J., \& Baras, R. (1995). Mother and infant activity and interaction in Israel and the United States: A comparative study. International Journal of Behavioral Development, 18, 63-82.

Bornstein, M. H., \& Tamis-LeMonda, C. S. (1989). Maternal responsiveness and cognitive development in children. In M. H. Bornstein (Ed.), Maternal responsiveness: Characteristics and consequences (pp. 49-62). San Francisco, CA: Jossey-Bass.

Bornstein, M. H., \& Tamis-LeMonda, C. S. (1997). Maternal responsiveness and infant mental abilities: Specific predictive relations. Infant Behavior and Development, 20, 283-296.

Bornstein, M. H., Tamis-LeMonda, C. S., Tal, J., Ludemann, P., Toda, S., Rahn, C.W., Pêcheux, M., Azuma, H., \& Vardi, D. (1992). Maternal responsiveness to infants in three societies: The United States, France, and Japan. Child Development, 63, 808-821.

Bowlby, J. (1984). Apego. São Paulo, SP: Martins Fontes.

Bussab, V. S. R. (1999). Da criança ao adulto - O que faz do ser humano o que ele é? In A. M. Carvalho (Ed.), O mundo social da criança: Natureza e cultura em ação (pp. 17-31). São Paulo, SP: Casa do Psicólogo.

Fantz, R. (1965). The origin of form perception. In P. H. Mussen, J. J. Conger \& J. Kagan (Eds.), Readings in child development and personality (pp. 72-84). New York: Harper \& Row.

Goldberg, S., Lojkasek, M., Gartner, G., \& Corter, C. (1989). In M. H. Bornstein (Ed.), Maternal responsiveness: Characteristics and consequences (pp. 89-103). San Francisco, CA: Jossey-Bass.

Greenfield, P. M. (2002). The mutual definition of culture and biology in development. In H. Keller, Y. H. Poortinga \& A. Shölmerich (Eds.), Between culture and biology (pp. 57-76). Cambridge, MA: Cambridge University Press.

Harkness, S. \& Super, C. M. (1992). Parental ethnoteories in action. In I. E. Sigel, A. V. McGillicuddy-DeLisi \& J. J. Goodnow (Eds.), Parental belief systems: The psychological consequences for children (pp. 373-391). Hillsdale, NJ: Lawrence Erlbaum.

Harkness, S., \& Super, C. M. (1994). Developmental niche: A theoretical framework for analyzing the household production of health. Social Science and Medicine, 38, 219-226.

Hewlett, B.S., Lamb, M. E., Shannon, D., Leyendecker, B., \& Schölmerich, A. (1998). Culture and early infancy among central african foragers and farmers. Developmental Psychology, 34, 653-661.

Keller, H. (1998, Nov.). The role of development for understanding the biological basis of cultural learning. Paper presented at the Symposium Theories of Individual Development: Demarcating and Integrating Metaperspectives, Wittenberg, Germany.

Keller, H. (2002a). Development as the interface between biology and culture: A conceptualization of early ontogenetic experiences. In H. Keller, Y. H. Poortinga \& A. Shölmerich (Eds.), Between culture and biology (pp. 215-240). Cambridge, MA: Cambridge University Press.

Keller, H. (2002b). Culture and development: Developmental pathways to individualism and interrelatedness. In W. J. Lonner, D. L. Dinnel, S.A. Hayes \& D. N. Sattler (Eds.), Online readings in Psychology and culture (Unit 11, Chapter 1). Retrieved from Center for Cross-Cultural Research, Western Washington University, from http://www.wwu.edu/ culture

Keller, H., Lohaus, A., Volker, S., Cappenberg, M., \& Chasiotis, A. (1999). Temporal contingency as an independent component of parenting behavior. Child Development. 70, 474485 . 
Ogbu, J. (1981). Origins of human competence: A culturalecological perspective. Child Development, 52, 413-429.

Ribas, A. F. P. (2004). Responsividade materna: Discussão conceitual, articulações teóricas e uma investigação empírica em contexto urbano brasileiro. Tese de Doutorado nãopublicada, Universidade do Estado do Rio de Janeiro, Rio de Janeiro, RJ

Ribas, A. F. P., Seidl de Moura, M. L., \& Ribas, R. C., Jr. (2003). Responsividade materna: Levantamento bibliográfico e discussão conceitual. Psicologia: Reflexão e Crítica, 16, 137-145.

Ribas, R. C., Jr., Seidl de Moura, M. L., Gomes, A. A. N., Soares, I. D., \& Bornstein, M. H. (2003). Socioeconomic status in Brazilian psychological research. Part 1: Validity, measurement, and application. Estudos de Psicologia, 8, 375-383.

Richman, A. L., Miller, P. M. \& LeVine, R. A. (1992). Cultural and educational variations in maternal responsiveness. Developmental Psychology, 28, 614-621.

Seidl de Moura, M. L. \& Ribas, A. F. P. (2004). Evidências sobre características de bebês recém-nascidos: Um convite a reflexões teóricas. In M. L. Seidl de Moura (Ed.), O bebê do século XXI e a psicologia em desenvolvimento (pp. 21-60). São Paulo, SP: Casa do Psicólogo.

Small, M. F. (1999). Our babies, ourselves. How biology and culture shape the way we parent. New York: Anchor Books.

Tamis-LeMonda, C. S., Bornstein, M. H., Baumwell, L., \& Damast, A. (1996). Responsive parenting in the second year: Specific influences on children's language and play. Early Development and Parenting, 5(4), 173-183.

Van Egeren, L. A., Barratt, M. S., \& Roach, M. A. (2001). Motherinfant responsiveness: Timing, mutual regulation, and interactional context. Developmental Psychology, 37, 684-697.

Wakschlag, L. S., \& Hans, S. L. (1999). Relation of maternal responsiveness during infancy to the development of behavior problems in high-risk youths. Developmental Psychology, 35, 569-579. 